\title{
Mechanically Driven Director-Switching of Liquid Crystal Elastomers Having Homeotropic Alignment
}

\author{
Ryo Mashita $^{1}$, Kenji Urayama ${ }^{1}$, Ichiro Kobayashi ${ }^{2}$ and Toshikazu Takigawa ${ }^{1}$ \\ 1. Department of Materials Chemistry, Kyoto University, Kyoto, Japan \\ 2. Electronic Materials Division, Nissan Chemicals, Funabashi, Japan
}

\begin{abstract}
We study the stretching driven director-switching in a thin film of liquid crystal elastomers having the initial director in the thickness direction ( $x$-direction). The thin film specimen is uniaxially stretched in the $z$-direction normal to the initial director. The stress-strain curve has the three characteristic regions: the small strain region (I) obeying linear elasticity, the moderate strain region (II) exhibiting a quasi-plateau stress, and the large strain region (III) where the stress increases again. The Poisson's ratio $\mu_{\mathrm{yz}}$ in the strain region (II) is considerably small $(\sim 0.17)$ while those in the region (I) and (III) are nearly 0.5 . These features in the strain region (II) result from the cooperative uniform rotation of director towards the stretching direction. The strain dependence of the IR dichroic ratio of the mesogen also supports this picture.
\end{abstract}

Key words: Liquid crystal elastomers, Liquid crystal polymers, Stretching, Poisson's ratio

\section{INTRODUCTION}

Liquid crystal elastomers (LCEs) have received much attention from the academic and industrial fields as a new class of materials.[1] LCEs are a cross of liquid crystals and elastomers, and they behave as a soft solid having liquid crystallinity without flowing because of cross-links. LCEs have a strong coupling of orientational order and rubber elasticity. The macroscopic shape of LCEs always reflects the orientational order of the constituent mesogenic molecules, and vice versa. The reversible switching of the nematic director in LCEs has been demonstrated by mechanical stress[2-5] and electric fields[6,7] normal to the initial director. In the earlier studies on mechanically induced director-switching, thin LCE films having a global director parallel to the plane surface (homogeneous alignment) were employed. In this case, an inhomogeneous texture for mesogen orientation in the middle stage of a $90^{\circ}$ switching was often observed.[8] This is because the global shear deformation of the specimen in the middle stage of director rotation is not possible owing to the clamping of both ends. Uniform director rota- tion is expected for a sufficiently thin LCE film having the initial director in the thickness direction (homeotropic alignment), because the contribution of shear deformation to overall deformation is negligible owing to the thin film geometry. In the present study, we study the directorswitching behavior of such LCE films under mechanical fields. The stress, Poisson's ratio and IR dichroic ratio of the constituent mesogens in the director-switching process are investigated as a function of the imposed strain.

\section{EXPERIMENTS}

\subsection{Sample}

A side chain type LCE was prepared by the photopolymerization of the reactive mesogenic monomer (Fig. 1) with 1,6-hexanediol diacryrate (cross-linker) in a glass cell whose surface was coated by a polyimide layer. This polyimide layer induces the mesogen to orient in the direction normal to the substrate surface. After the crosslinking reaction, the resultant film was carefully separated from the substrates, and was allowed to swell in dichloromethane to wash out the 
unreacted molecules. A $50 \propto \mathrm{m}$-thick LCE film having the director in the thickness direction was obtained by drying the deswollen film. The nematic-isotropic transition temperauture was 96 ${ }^{\circ} \mathrm{C}$. The glass transion temperature was around 50 ${ }^{\circ} \mathrm{C}$.

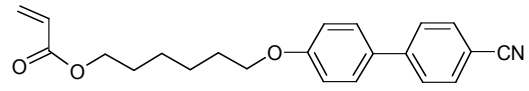

Fig. 1. Molecular structure of the pendant mesogen

\subsection{Measurement}

A film specimen $(10 \mathrm{~mm} \times 2 \mathrm{~mm} \times 50 \propto \mathrm{m})$ was stretched in the $z$-direction normal to the initial director ( $x$-direction) at a constant crosshead speed (Figure 2). The crosshead speed was varied from 0.5 to $50 \mathrm{~mm} / \mathrm{min}$. The stretching process was recorded by a video camera to examine the dimensional changes in the $y$ - and $z$-directions.

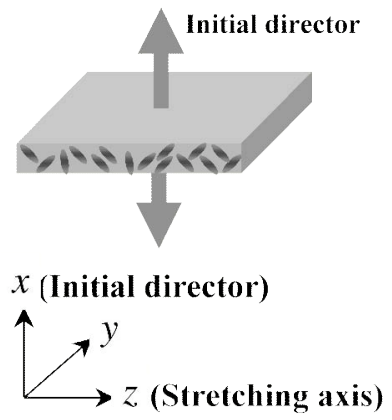

Fig. 2. Initial director and stretching direction.

The FT-IR measurement during the stretching was separately conducted using the same specimen as that in the stress-strain measurement. The absorbance of the cyano group $(A)$ at the end of the mesogen (Fig. 1) was measured using the incident beam polarized in the directions along $(z)$ and normal $(y)$ to the stretching axis. The dichroic ratio $D_{\mathrm{z}, \mathrm{y}}=A_{\mathrm{z}} / A_{\mathrm{y}}$ gives us the information about the orientational order of the mesogen. The specimen was elongated stepwise by $5 \%$ in strain. The average strain rate in the whole FT-IR measurement (ca. $0.1 \mathrm{~mm} / \mathrm{min}$ ) was in the same order of the slowest one in the tensile measurement with a constant crosshead speed.

\section{RESULTS AND DISCUSSIONS}

Figure 3 shows the $T$ dependence of the nominal $\operatorname{stress}\left(\sigma_{\mathrm{z}}\right)$-true $\operatorname{strain}\left(\varepsilon_{\mathrm{z}}\right)$ curves at a cross-head speed of $0.5 \mathrm{~mm} / \mathrm{min}$. In the isotropic state of $T>$ $T_{\mathrm{NI}}$, the shape of the $\sigma_{\mathrm{z}}-\varepsilon_{\mathrm{x}}$ curve is similar to that of conventional rubbers. The LCE in the isotropic state behaves as usual crosslinked rubbers. In contrast, in the nematic state of $T<T_{\mathrm{NI}}$, the $\sigma_{\mathrm{z}}-\varepsilon_{\mathrm{z}}$ curves have a quasi-plateau region in the moderate strain range. The quasi-plateau region becomes broader with decreasing $T$. They have a linear elasticity region at small deformations. The strains beyond the quasi-plateau region lead to an increase in stress again.

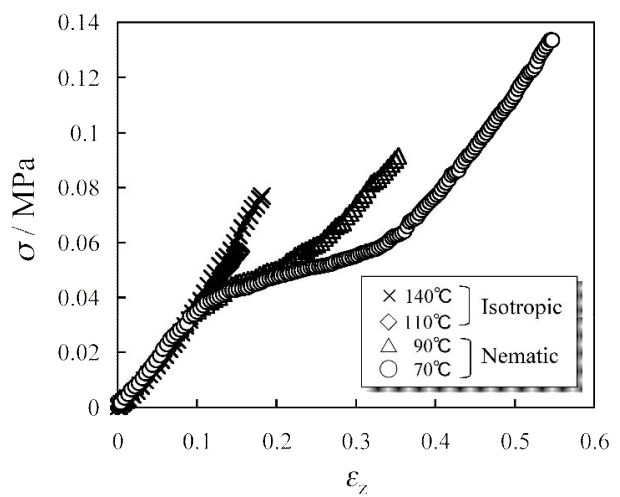

Fig. 3. Temperature dependence of $\sigma_{\mathrm{z}}-\varepsilon_{\mathrm{z}}$ curves at a cross-head speed of $0.5 \mathrm{~mm} / \mathrm{min}$.

Figure 4 shows the relation between $\varepsilon_{\mathrm{z}}$ and $\varepsilon_{\mathrm{y}}$ during stretching in the isotropic and nematic states. In the isotropic state, all data points fall on a straight line with a slope of 0.5 . The slope corresponds to the Poisson's ratio $\left(\propto_{y z}\right)$. In the isotropic phase, $\propto_{y z}$ is the same as that of incompressible conventional rubbers. In the nematic state, the slopes at the small deformations of $\varepsilon_{\mathrm{z}}<0.18$ and the large strains of $\varepsilon_{\mathrm{x}}>0.32$ are well approximated by 0.5 . However, the slope $(\sim 0.17)$ in the moderate strains of $0.18<\varepsilon_{\mathrm{x}}<0.32$ is definitely smaller than 0.5 . In this region, the elongation-induced shrinkage in the $y$-direction is suppressed. Instead, the corresponding thickness change in the $z$ direction becomes larger owing to the volume constancy. It should be noted that the strain region 


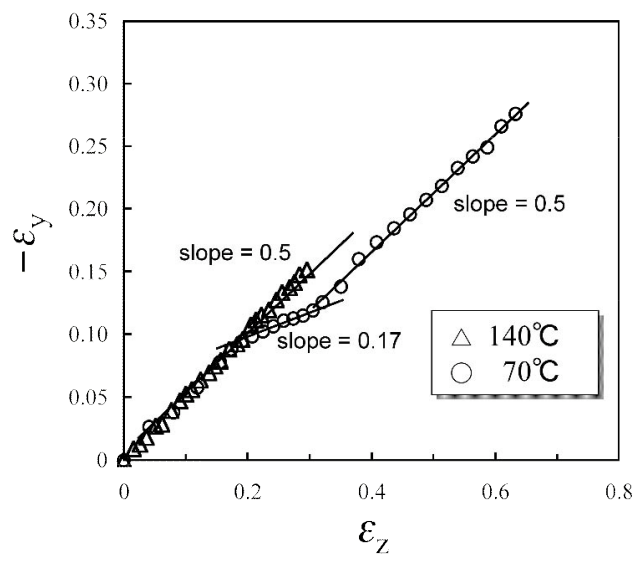

Fig. 4. The $\varepsilon_{\mathrm{y}}-\varepsilon_{\mathrm{z}}$ relations in the isotropic and nematic states at a cross-head speed of $0.5 \mathrm{~mm} / \mathrm{min}$. The slope corresponds to the Poisson's ratio $\propto_{\mathrm{yz}}$.

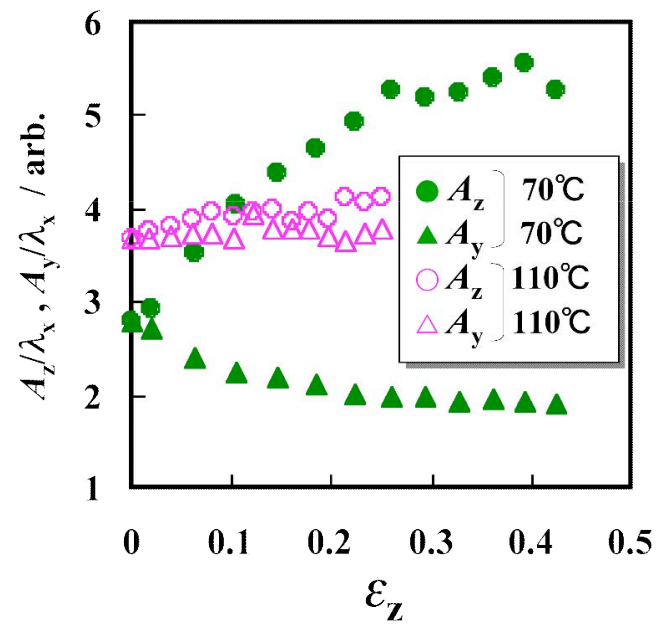

Fig. 5. The $\varepsilon_{\mathrm{z}}$ dependence of $A_{\mathrm{y}}$ and $A_{\mathrm{z}}$ in the isotropic and nematic states.

having a small value of $\propto_{y z}$ is almost identical with that showing a quasi-plateau stress in Figure 3.

Figure 5 shows the $\varepsilon_{\mathrm{z}}$ dependence of $A_{\mathrm{y}}$ and $A_{\mathrm{z}}$. The absorbance at each strain was reduced by $\lambda_{z}$ to consider the effective thickness change. In the isotropic state $\left(110^{\circ} \mathrm{C}\right)$, the absorbance is not significantly influenced by elongation. The orientation of the dangling mesogens remains almost random under elongation. In contrast, in the nematic state, the stretching results in an increase in $A_{\mathrm{z}}$ and decrease in $A_{\mathrm{y}}$. They become constant at the large strains of $\varepsilon_{\mathrm{z}}>0.32$. This clearly indicates that the elongation drives the mesogen reorientation in the stretching direction. Importantly, $A_{\mathrm{y}}$ and $A_{\mathrm{z}}$ saturates around the strain at which the stress starts to increase again (i.e., the upper end of the quasi-plateau region), and no growth of $A$ takes place at the high strains of $\varepsilon_{\mathrm{z}}>0.32$. The mesogen reorientation completes at $\varepsilon_{\mathrm{z}} \approx 0.32$. The dichroic ratio $A_{\mathrm{z}} / A_{\mathrm{y}}$ in the steady state corresponds to ca. 0.38 in the orientational order parameter. The stress upturn at high strains stems from the stretching of network chains as observed in usual crosslinked rubbers. No significant hysteresis was observed for the $\sigma_{\mathrm{z}}-\varepsilon_{\mathrm{z}}$ and $A-\varepsilon_{\mathrm{z}}$ relations, although the data in the reverse process are not shown here.

These results suggest the following scenario for the process of the mesogen reorientation driven by stretching. The small $\propto_{y z}$ in the region (II) of $0.1<$ $\varepsilon_{\mathrm{z}}<0.32$ means that the deformation dominantly occurs in the $x-z$ plane where the director rotates. This deformation mode is similar to pure shear $\left(\propto_{\mathrm{yz}}=0\right.$ and $\varepsilon_{\mathrm{y}}=0$ ) rather than uniaxial stretching. The similar deformation mode was observed in the director-switching by electric fields.[7] The quasiplateau stress in the region (II) indicates that the uniform director rotation readily proceeds by a small stretching force. In the region (III) of $\varepsilon_{z}>$ 0.32 after the completion of director reorientation ( $A=$ const.), the mechanical behavior is similar to that of conventional elastomers. In the small strain region (I) of $\varepsilon_{z}<0.1$, a finite change in $A$ is also observed, while the mechanical behaviors $\left(\sigma_{\mathrm{z}}-\varepsilon_{\mathrm{z}}\right.$ curve and $\propto_{\mathrm{yz}}$ ) are the same as those of typical elastomers. This implies that a finite nematic order in the stretching direction is needed to induce the cooperative director rotation observed in the region II. In fact, $A_{\mathrm{y}}$ appreciably changes with $\varepsilon_{\mathrm{z}}$ in the region I. This means that a mesogen reorientation in the $y-z$ plane occurs in addition to that in the $x-z$ plane, because $A_{\mathrm{y}}$ must be constant if the mesogen reorientation takes place only in the $x-z$ plane.

\section{CONCLUSIONS}

The initial homeotropic mesogen alignment in a thin LCE film can be switched by a small uniaxial stretching stress to the homogeneous alignment along the elongation axis. The stress-strain curve exhibits a quasi-plateau at the strain region where the cooperative mesogen reorientation takes place. 
In this strain region, the dimensional change in the direction irrelevant to the director rotation is significantly suppressed, and the dominant distortion occurs in the plane regarding the director rotation. This deformation mode is similar to pure shear, rather than uniaxial stretching. The stress upturns again, and the usual uniaxial stretching mode appears at the large strains where the director reorientation in the stretching direction is completed.

\section{REFERENCES}

1. M. Warner and E. Terentjev, Liquid Crystals Elastomers, Cambridge University Press, Cambridge (2003).

2. G. R. Mitchell, F. J. Davis and W. Guo, Phys. Rev. Lett., 71 (1993), pp. 2947.

3. J. Kupfer and H. Finkelmann, Macromol. Chem. Phys., 195 (1994), pp. 1353.

4. H. Finkelmann, I. Kundler, E. M. Terentjev and M. Warner, J. Phys. II, 7 (1997), pp. 1059.

5. F. Zhang, P. A. Heiney, A. Srinivsan, J. Naciri and B. Ratna, Phy. Rev. E, 73 (2006), pp. 021701 .

6. K. Urayama, S. Honda and T. Takigawa, Macromolecules, 38 (2005), pp. 3574.

7. K. Urayama, S. Honda and T. Takigawa, Macromolecules, 39 (2006), pp. 1943.

8. I. Kundler and H. Finkelmann, Macromol.
Chem. Rapid Commun. 16 (1995), pp. 6679. 